\title{
DISTINGUISHING FEATURES OF PHASE TRANSFORMATION IN THE SURFACE LAYER OF HARDENED STEEL WITH $(\alpha+\gamma)$-TWO-PHASE STRUCTURE DURING PLASTO-ELASTIC LOADING AT DIFFERENT RATES
}

\author{
L.E. Alerseeva, V.A. Sinelnikov and G.A. Filippov \\ I.P. Bardin State Scientific Centre \\ 107005 Moscow, Russia
}

\begin{abstract}
An effect of loading during uniaxial tension at different rates ranging from $10^{-4}$ to $10^{-2} \mathrm{~s}^{-1}$ on the phase transformation "austenite-martensite" in the surface layer of hardened $0.36 \% \mathrm{C}-14 \% \mathrm{Ni}$ steel type with an unbalanced $(\alpha+\gamma)$-structure has been determined by an X-ray structure analysis. The mechanisms of $(\gamma-\alpha)$-transformation through the depth of steel surface layer and character of this transformation propagation along the specimen length depending on external stress value and tension rate have been defined.
\end{abstract}

PACS numbers: $62.20 .-\mathrm{x}$

\section{Introduction and experimental technique}

A structural state of a hardened steel surface layer during uniaxial tension within an elastic range and small deformations are scantily known. It is a matter of general experience that plastic deformation causes the phase transformation of austenite into martensite $(\gamma-\alpha)$. The martensitic transformation becomes energetically advantageous since a thermodynamic potential in the field of external forces decreases [1]. During loading in an elastic range internal stresses are added to external ones in places of its local concentration. If the effective stress has reached the yield strength value, a microplastic deformation can occur [2]. This deformation is likely to initiate a $(\gamma-\alpha)$ - transformation in steel. The surface layer becomes faulty because defects are concentrated precisely at the surface lattice to the greatest extent. Moreover, different mechanical microdefects take place here. All above-mentioned facts can effect the deformation distribution in a specimen depth and length during uniaxial tension in elastic and plastic regions.

It was desired to estimate the influence of loading in the elasto-plastic region on the character of the $(\gamma-\alpha)$-transformation in a steel surface layer. 
Hardened $0.36 \% \mathrm{C}-14 \% \mathrm{Ni}$ steel (hardening at $780^{\circ} \mathrm{C}$, air cooling) with unbalanced structure, $80 \%$ of martensite $(M)+20 \%$ of retained austenite $\left(A_{\mathrm{r}}\right)$, was the subject of this investigation.

The test specimens of size $3.5 \times 7 \times 145 \mathrm{~mm}$ were loaded at uniaxial tension with different rates $\left(10^{-4}-10^{-2} \mathrm{~s}^{-1}\right)$ in the following way: up to the stress values of elastic range ( $\left.\sigma_{k}=0.7 \sigma_{0.2}\right)$; up to the ultimate strength value $\left(\sigma_{\mathrm{b}}\right)$; and up to the neck appearance (Instrong machine). Different values of a tension rate ha ve allowed one to take account of the time factor. The $(\gamma-\alpha)$-transformation proceeding was judged from an decrease in $A_{\mathrm{r}}$ amount. $A_{\mathrm{r}}$ quantity was determined by an X-ray structure analysis. The experiment was performed at a diffractometer DRON-3 (Fe-irr.) of fixed areas of a surface in the transverse direction with $10 \mathrm{~mm}$ step along an operating length $(100 \mathrm{~mm})$, in a depth range of $60-120 \mu \mathrm{m}$ on the samples with the pre- and post-pickling treatment.

\section{Experimental results and discussion}

As the result of the experiments the pattern of $A_{\mathrm{r}}$ distribution over the surface of specimens along its operating length before and after loading was obtained. It was found that after the plastic deformation up to the failure at the rates of $\varepsilon=10^{-2}, 10^{-3}, 10^{-4} \mathrm{~s}^{-1}$ the $(\gamma-\alpha)$-phase transition along the operating length occurs $\left(A_{\mathrm{r}}\right.$ quantity decreases drastically - from 20 to $8 \%$ - as a consequence of its transformation into martensite, in comparison with an unloaded state). In the process the $A_{\mathrm{r}}$ distribution along the specimen length is uniform excepting the breakpoint, where the $(\gamma-\alpha)$-transformation is very nearly equal to $100 \%$.

Another pattern is observed during small plastic deformations and deformations in the elastic zone. Loading to the stress magnitudes of $0.7 \sigma_{0.2}\left(\varepsilon=10^{-2}\right.$, $10^{-5} \mathrm{~s}^{-1}$ ) (Fig. 1) also leads to the phase transition-partial transformation of $A_{\mathrm{r}}$ into $M$. ( $A_{\mathrm{r}}$ amount reduces from $20 \%$ in an unloaded state to $13 \%$ after loading to $\left.0.7 \sigma_{0.2}\right)$. As opposed to the unloaded state, in this case $A_{\mathbf{r}}$ distribution along the specimen length is inhomogeneous and wave-like ( 2 minima are observed). With the strain rate the decline amount of martensite increases, intensification of the phase transition and a rise in $A_{\mathrm{r}}$ distribution inhomogeneity along the specimen length take place. As a result two minima degenerate into a single, deeper one. The intensity of the $(\gamma-\alpha)$-transformation grows when stress values increase up to $\sigma_{\mathrm{b}}\left(A_{\mathbf{r}}\right.$ amount decreases more drastically - from 20 to $\left.6 \%\right)$. In this case inhomogeneity of $A_{\mathrm{r}}$ distribution increases also with a rise of the tension value and reduction of the deformation rate, as in the case of loading to $0.7 \sigma_{0.2}$. But the maximum amplitude of $A_{\mathrm{r}}$ variation with the strain rate decrease from $10^{-2}$ to $10^{-4} \mathrm{~s}^{-1}$ is considerably greater than at $0.7 \sigma_{0.2}$ (from 20 to $0 \%$ ). Referring to Fig. 1, in either case the $A_{\mathrm{r}}$ distribution curve has two minima, degenerating into a single, deeper one, with $\varepsilon$ decrease to $10^{-4} \mathrm{~s}^{-1}$. In the zone corresponding to $A_{\mathrm{r}}$ a minimum during reloading specimen break occurs.

An X-ray structure analysis of specimen surface after removal of a $60 \mu \mathrm{m}$ thick layer by means of electrolytic etching allowed one to determine that the $(\gamma-\alpha)$-transformation and inhomogeneity of $A_{\mathrm{r}}$ distribution along the specimen length at loading within the elastic zone up to $0.7 \sigma_{0.2}$ is observed only in a thin surface layer - less thick than $60 \mu \mathrm{m}$ (Fig. 2). At a depth of $60 \mu \mathrm{m}$ the phase 

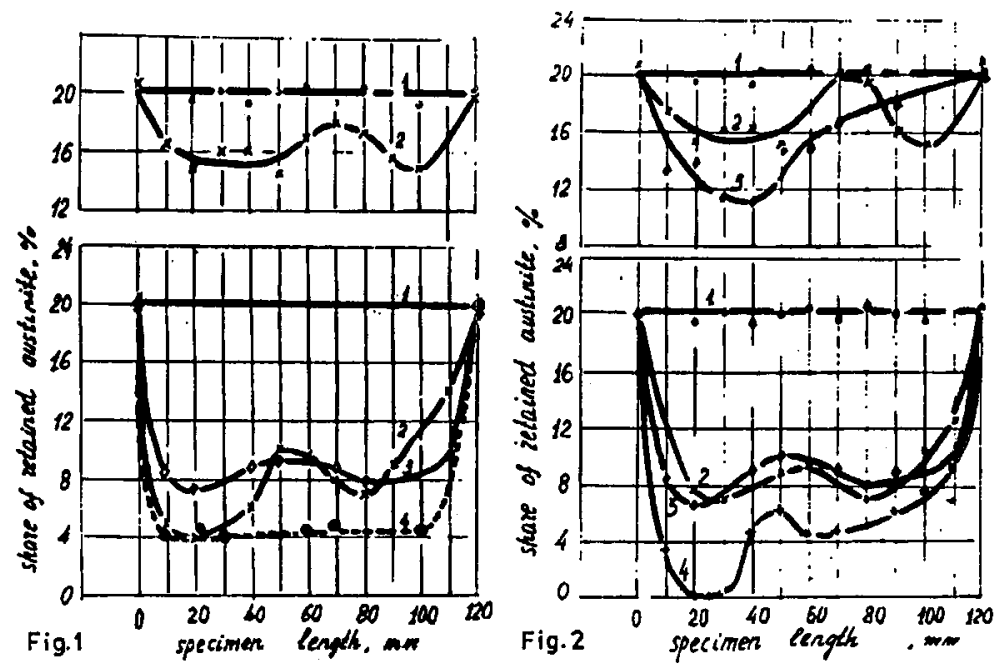

Fig. 1. Variation of retained austenite amount $A_{\mathrm{r}}$ along the specimen length as a result of loading up to the values of $0.7 \sigma_{0.2}$ (upper part) and $\sigma_{\mathrm{b}}$ (lower part) with the strain rates of $\varepsilon=10^{-2}(2) ; 10^{-3}(3) ; 10^{-4}(4) \mathrm{s}^{-1}$. (1) - initial pre-loaded state.

Fig. 2 Variation of retained austenite amount $A_{\mathrm{r}}$ along the specimen length as a result of loading up to the values of $0.7 \sigma_{0.2}$ (upper part) and $\sigma_{\mathrm{b}}$ (lower part) with the strain rates of $\varepsilon=10^{-2}(2) ; 10^{-3}(3) ; 10^{-4}(4) \mathrm{s}^{-1}$ after removal of a $60 \mathrm{mc}$ thick layer. (1) - initial pre-loaded state.

transformation is absent, $A_{\mathrm{r}}$ amount and its distribution correspond to the unloaded state. But as a tension value increases up to $\sigma_{\mathrm{b}}$, the $(\gamma-\alpha)$-transformation can be observed at a depth of $60 \mu \mathrm{m}$ (Fig. 2), where inhomogeneous $A_{\mathrm{r}}$ distribution is retained at $\varepsilon=10^{-2}$ and $10^{-3} \mathrm{~s}^{-1}$, and if $\varepsilon=10^{-4} \mathrm{~s}^{-1}$ this distribution becomes uniform along the whole specimen length. Subsequent investigations have shown that a thickness of the layer, in which the $(\gamma-\alpha)$-transformation occurs at loading up to $\sigma_{\mathrm{b}}$, is $120 \mu \mathrm{m}$.

It was determined by means of the X-ray structure analysis of $\alpha$-phase before loading, after loading up to $0.7 \sigma_{0.2}$ and $\sigma_{\mathrm{b}}$, and after specimen break that the deformation causes a relaxation of residual microstresses in martensite via the microplastic deformation. This is reflected in a decrease in the (211) reflex width, especially sharp during loading in the macroelastic region as compared with the initial pre-loaded state.

The $(\gamma-\alpha)$-phase transformation during loading in the macroelastic zone is caused by the microplastic deformation. The relationship between the $(\gamma-\alpha)$-transformation and the deformation rate is connected with the isothermic character of the martensitic transformation in $0.36 \% \mathrm{C}-14 \% \mathrm{Ni}$ steel type.

Inhomogeneity of the phase transformation in the steel surface layer can be associated with the fact that the diffusionless character of the martensitic transformation initiates high internal microstresses distributed inhomogeneously. These 
microstresses are an additional stress source in the fault surface layer and lead to the thermodynamic balance disruption in (to) the field of external forces.

\section{Conclusions}

1. During loading of hardened steel specimens to the values below the yield strength (uniaxial tension) phase the $(\gamma-\alpha)$-transformation occurs only in a thin layer less thick than $60 \mu \mathrm{m}$. With an external stress increasing up to $\sigma_{\mathrm{b}}$ and/or a strain rate decreasing, this layer thickness and intensity of the $(\gamma-\alpha)$-transformation grow.

2. At loading in the plasto-elastic region a distribution of retained austenite $A_{\mathbf{r}}$ in a steel surface layer along the specimen length is inhomogeneous and wave-like. Inhomogeneity of the $(\gamma-\alpha)$-transformation depends on a value of external stress and the strain rate. At greater deformations (up to break) $A_{\mathrm{r}}$ distribution is uniform along the specimen length.

3. At uniaxial tension of hardened steel in the elastic and plastic region the relaxation of residual microstresses takes place.

\section{References}

[1] M.N. Pankova, A.L. Roitburd, Fiz. Met. Metalloved. 58, 716 (1984).

[2] A.R. Marder, A.O. Bencoper, G. Krauss, Trans. AIME 1, 15 (1970). 